\title{
Invitation to submit titles of books for review
}

(C) Springer Science+Business Media B.V. \& International Society for Plant Pathology 2010

Readers and publishers are invited to submit the titles of books appropriate for review in Food Security. Please send details, including, in addition to the full title, the name(s) of the author(s)/editor(s), the name of the publisher and the ISBN to:

Professor David Ingram

Food Security Book Reviews Editor

Centre for Sustainable Agriculture

The Lancaster Environment Centre, Lancaster University

Lancaster LA1 4YQ, UK (Email: d.ingram@lancaster.ac.uk) 